\title{
Fatores associados ao sucesso da ventilação não invasiva em crianças com insuficiência respiratória aguda em situação de emergência
}

\author{
Factors associated with the success of non-invasive ventilation in children with acute res- \\ piratory failure in an emergency
}
Factores asociados con el éxito de la ventilación no invasiva en niños con insuficiencia respiratoria aguda en una emergencia

Cinthia Torres Leite ${ }^{1 *}$, Maria de Fatima Pombo Sant'Anna², Clemax Couto Sant'Anna ${ }^{3}$, Maria Luiza Guerra de Toledo ${ }^{4}$.

\section{RESUMO}

Objetivo: Avaliar o uso da ventilação não invasiva (VNI) por pressão positiva em crianças com insuficiência respiratória aguda (IRA) internadas em emergência. Métodos: Estudo longitudinal, retrospectivo, analítico. Coleta de dados de prontuários (1 de julho de 2014 a 30 de outubro de 2016). Estudaram-se variáveis demográficas e clínicas e o desfecho dos pacientes: positivo (macronebulizacão) ou negativo (ventilação mecânica invasiva). Foi empregada a escala de Wood Downes (EWD) para insuficiência respiratória. Utilizada estatística descritiva e regressão logística univariada e multivariada para estudar as variáveis relacionadas ao desfecho. Resultados: Havia 27(51\%) pacientes do sexo feminino; 21(39,6\%) com bronquiolite; 20 $(37,7 \%)$ com pneumonia e $12(22,7 \%)$ com asma. Observou-se que quanto menor a idade maior a probabilidade de desfecho negativo $(p<0,001)$. Para cada aumento de 1 unidade na EWD pré VNI, o risco de desfecho positivo diminuiu em 75\%. A cada aumento de 1 unidade na saturação de O2(Sat O2) pós VNI, o risco de desfecho positivo aumentou três vezes seu valor $(p<0,001)$. Conclusão: Avaliou-se que o uso da VNI em crianças com IRA foi influenciado negativamente pela baixa idade e pelo aumento na EWD e positivamente pelo aumento da Sat O2 pós VNI.

Palavras- chave: Insuficiência respiratória, Ventilação não invasiva, Criança.

\begin{abstract}
Objective: To evaluate the use of non-invasive positive pressure ventilation (NIV) in children with acute respiratory failure (ARI) admitted to an emergency room. Methods: Longitudinal, retrospective, analytical study. Collection of data from medical records (July 1, 2014 to October 30, 2016). Demographic and clinical variables and patient outcomes were studied: positive (macronebulization) or negative (invasive mechanical ventilation). The Wood Downes scale (EWD) was used for respiratory failure. Descriptive statistics and univariate and multivariate logistic regression were used to study the variables related to the outcome. Results: There were 27 (51\%) female patients; 21 (39.6\%) with bronchiolitis; 20 (37.7\%) with pneumonia and $12(22.7 \%)$ with asthma. It was observed that the younger the age, the greater the probability of a negative outcome $(p<0.001)$. For each 1 -unit increase in pre-NIV EWD, the risk of a positive outcome decreased by $75 \%$. With each 1 -unit increase in $\mathrm{O} 2$ saturation (Sat O2) after NIV, the risk of a positive outcome increased three times its value ( $p$ $<0.001)$. Conclusion: It was assessed that the use of NIV was negatively influenced by young age and by an increase in EWD and positively by an increase in Sat O2 after NIV.
\end{abstract}

Keywords: Respiratory insufficiency, Noninvasive ventilation, Child.

\footnotetext{
1 Universidade do Rio de Janeiro (UNIRIO), Rio de Janeiro - RJ. *E-mail: cinleite@hotmail.com

2 Universidade Federal Fluminense(UFF), Rio de Janeiro - RJ.

3 Universidade Federal do Rio de Janeiro (UFRJ), Rio de Janeiro - RJ.

4 Instituto Brasileiro de Geografia e Estatística (IBGE), Rio de Janeiro - RJ.
} 


\section{RESUMEN}

Objetivo: Evaluar el uso de ventilación no invasiva (VNI) con presión positiva en niños con insuficiencia respiratoria aguda (IRA) ingresados en urgencias. Métodos: Estudio longitudinal, retrospectivo y analítico. Recolección de datos de historias clínicas (1 de julio de 2014 al 30 de octubre de 2016). Se estudiaron variables demográficas, clínicas y resultados de los pacientes: positivos (macronebulización) o negativos (ventilación mecánica invasiva). Para la insuficiencia respiratoria se utilizó la escala Wood Downes (EWD). Se utilizó estadística descriptiva y regresión logística univariante y multivariante para estudiar las variables relacionadas con el resultado. Resultados: Hubo 27 (51\%) pacientes del sexo femenino; 21 (39,6\%) con bronquiolitis; 20 $(37,7 \%)$ con neumonía y $12(22,7 \%)$ con asma. Se observó que, a menor edad, mayor probabilidad de resultado negativo $(p<0,001)$. Por cada aumento de 1 unidad en la EWD anterior a la VNI, el riesgo de un resultado positivo ha disminuido en un $75 \%$. Con cada aumento de 1 unidad en la saturación de O2 (Sat O2) después de la VNI, el riesgo de un resultado positivo aumentó tres veces en valor $(p<0,001)$. Conclusión: Se evaluó que el uso de la VNI se vio influido negativamente por la edad temprana y por un aumento en la EWD y positivamente por un aumento en la Sat O2 después de la VNI.

Palabras clave: Insuficiencia respiratoria, Ventilación no invasiva, Niño.

\section{INTRODUÇÃO}

A insuficiência respiratória aguda (IRA) é uma das afecções mais frequentes na emergência pediátrica, com grande potencial de morbi-mortalidade infantil. As emergências respiratórias pediátricas estão entre as causas mais frequentes de internação hospitalar e óbitos em crianças menores de 1 ano de idade (HOLANDA MA, et al., 2001; SOCIEDADE BRASILEIRA DE PEDIATRIA, 2017; HAMMER J, 2013). A população pediátrica é suscetível a desenvolver IRA grave em decorrência de peculiaridades anatômicas e características fisiológicas e imunológicas (SOCIEDADE BRASILEIRA DE PEDIATRIA, 2017).

Dentre as condições anatomo-fisiológicas das crianças que podem contribuir para a IRA estão: o maior metabolismo e maior consumo de O2; a língua em tamanho maior que o normal, próxima ao palato mole podendo ocorrer a obstrução da via aérea; a ventilação colateral (canais de Lambert e Poros de Kohn) ainda bem pouco desenvolvidos ou ausentes, favorecendo a formação de atelectasias; o diafragma perpendicular ao tórax e caixa torácica mais complacente ocasionando a incoordenação toraco- abdominal principalmente no no sono REM (rapid moviment eyes- movimento rápido dos olhos), o pequeno diâmetro das vias aéreas com tendência à obstrução; o tórax em barril diminuindo e dificultando os movimentos compensatórios para aumentar o volume corrente; os pulmões com menos elastina nas crianças pequenas, ocasionando diminuição na propriedade de recolhimento elástico, com consequente diminuição na complacência pulmonar e a musculatura respiratória menos desenvolvida e consequentemente frequências respiratórias mais elevadas (FONSECA JG, et al., 2013). Por todos esses motivos, em vigência de afecção respiratória ou qualquer condição que ocasione e evolua para IRA, principalmente no lactente, primeira infância ou pacientes com menor idade, faz-se necessário muitas vezes o auxilio de suporte ventilatório assim como oxigenioterapia, em escolha inicial quando as condições do paciente indicarem o uso.

A oxigenoterapia, ofertada por cateter nasal ou outros dispositivos, é um importante recurso terapêutico para pacientes com IRA e consegue manter a estabilidade do padrão respiratório. A oferta de oxigênio (O2) é mandatória na IRA, mesmo com saturação de O2 (SatO2) >92\%, para otimizar o transporte de O2 aos tecidos e diminuir o trabalho respiratório melhorando o volume minuto (Vol Min) (SOCIEDADE BRASILEIRA DE PEDIATRIA, 2017).

A ventilação não invasiva (VNI) na IRA permite recrutar alvéolos colapsados ou não ventilados, aumentar a capacidade residual funcional, melhorar a relação ventilação-perfusão, optimizar a dinâmica respiratória, diminuir o trabalho respiratório e melhorar o intercâmbio gasoso (MUÑOZ-BONET JL, et al., 2010; RIMENSBERGER PC, 2000). A VNI provê assistência ventilatória sem necessidade de via aérea artificial. É cada vez mais aceita e empregada em casos de IRA em geral (SCHNEIDER J e SWEBERB T, 2013; SMITH A, et al., 2019; POPAT B e JONES AT, 2012). 
Há uma parcela de pacientes com IRA que evoluem para intubação traqueal e ventilação mecânica para auxiliar na correção da hipoxemia, hipoventilação e melhora do desconforto respiratório. No entanto, a intubação traqueal pode acarretar em varias complicações como PAV (pneumonia associada a ventilação mecânica), aumento da necessidade do uso de sedativos e aumento no tempo de internação hospitalar, podendo contribuir com fatores adversos e maiores gastos (TOBIN MJ, 2000).

A elevada incidência e a grande variedade de complicações ocasionadas devido a utilização da ventilação mecânica invasiva no tratamento da IRA, entre elas a PAV e a lesão pulmonar induzida por este procedimento, foram os principais fatores que impulsionaram o surgimento de excelentes estudos sobre o uso da ventilação não-invasiva. (GONZAGA SC, et al., 2011).

Dado o crescente aumento de pacientes nas emergências pediátricas em decorrência do perfil sazonal das doenças e das novas afecções existentes fez se necessário observar a prática clínica com possibilidade de ampliar avanços terapêuticos através de nosso estudo facilitando a qualidade de atendimentos e a sobrevida de tais pacientes. Este estudo visou avaliar o uso da VNI em crianças com IRA internadas em emergência, destacando bronquiolite viral aguda (BVA), asma e pneumonia adquirida na comunidade (PAC).

Baseado em estudos anteriormente publicados, tendo em vista a necessidade de ampliar conhecimentos nesta área e melhorar as condições evolutivas das crianças que dão entrada a emergencia em angustia respiratória por IRA, se faz necessário a adequada intervenção da VNI, especificamente, para estabilização do quadro de desconforto respiratório de modo a buscar a boa evolução clínica desde o inicio da doença, visando oportunidade de melhora com menor tempo de internação, maior comodidade, diminuindo comorbidades e facilitando o fluxo de atendimentos e altas hospitalares.

\section{MÉTODOS}

Foi realizado estudo retrospectivo, longitudinal, descritivo no setor de emergência pediátrica de um hospital de alta complexidade, no período de $1^{\circ}$ de julho de 2014 a 30 de outubro de 2016 . O hospital tem em média 2.398 internações em emergência pediátrica a cada semestre.

Foram estudadas variáveis demográficas: sexo, idade e cor; variáveis clínicas: data inicial da VNI, data final da VNI, sinais vitais (freqüência respiratória, freqüência cardíaca, saturação de oxigênio); tipo de interface; modo ventilatório e parâmetros da VNI; data de alta hospitalar; desfecho positivo (pós VNI evolução para macronebulizacão/ ar ambiente) e desfecho negativo (pós VNI evolução para ventilação mecânica invasiva); variáveis de internação: data de internação, diagnóstico, escala de insuficiência respiratória com emprego da escala de Wood Downes (EWD) variando entre leve, moderada e grave; observada antes e após a VNI.

Foram incluídos pacientes, com idades entre 1 mês e 12 anos e 11 meses com diagnóstico de IRA assistidos por VNI por pressão positiva e excluídos pacientes com IRA de elevada gravidade com evolução imediata para ventilação mecânica invasiva. Realizada coleta de dados através de ficha clínica, onde eram incluídas as variáveis analisadas. Pacientes indicados a VNI eram avaliados por equipe multidisciplinar, através do parecer médico e fisioterapêutico.

A EWD consiste na avaliação da IRA contabilizando sinais vitais e padrão respiratório com variação de leve, moderado e grave. Foi aplicada antes e após o uso da VNI instituída pelo Sistema de ventilação Servo S.10 As análises estatísticas foram realizadas com o software $R$ versão 3.6.0.

As variáveis categóricas foram expressas em termos de frequências absolutas e relativas, e variáveis numéricas foram expressas por medianas e amplitudes inter-quartílicas (AIQ). Análises univariadas e multivariadas para fatores associados ao desfecho positivo foram realizadas. $\mathrm{Na}$ análise univariada, dados categóricos foram comparados utilizando-se tabelas de contingência e teste qui-quadrado; variáveis numéricas foram comparadas utilizando-se o teste $U$ Mann-Whitney. A análise multivariada foi conduzida utilizando-se modelos de regressão logística binária com eliminação de variáveis. Todos os valores-p menores do que 0,05 foram considerados estatisticamente significativos. A qualidade do ajuste da regressão logística foi avaliada pelo teste de Hosmer-Lemeshow. 


\section{RESULTADOS}

Foram incluídos no estudo 53 pacientes. Destaca-se que houve predomínio de EWD 4, pós aplicação de VNI, demonstrando grau de insuficiência respiratória leve enquanto que no EWD pré VNI houve predomínio de grau de insuficiência respiratória grave, demonstrando que na admissão, essas crianças em sua maioria encontravam-se graves em relação ao desconforto respiratório, apresentando melhora após aplicação da VNI. Desta forma, a maior parte apresentou desfecho positivo (81,1\%) (Figura 1).

Figura 1 - Fluxograma do desfecho dos paciente no estudo.

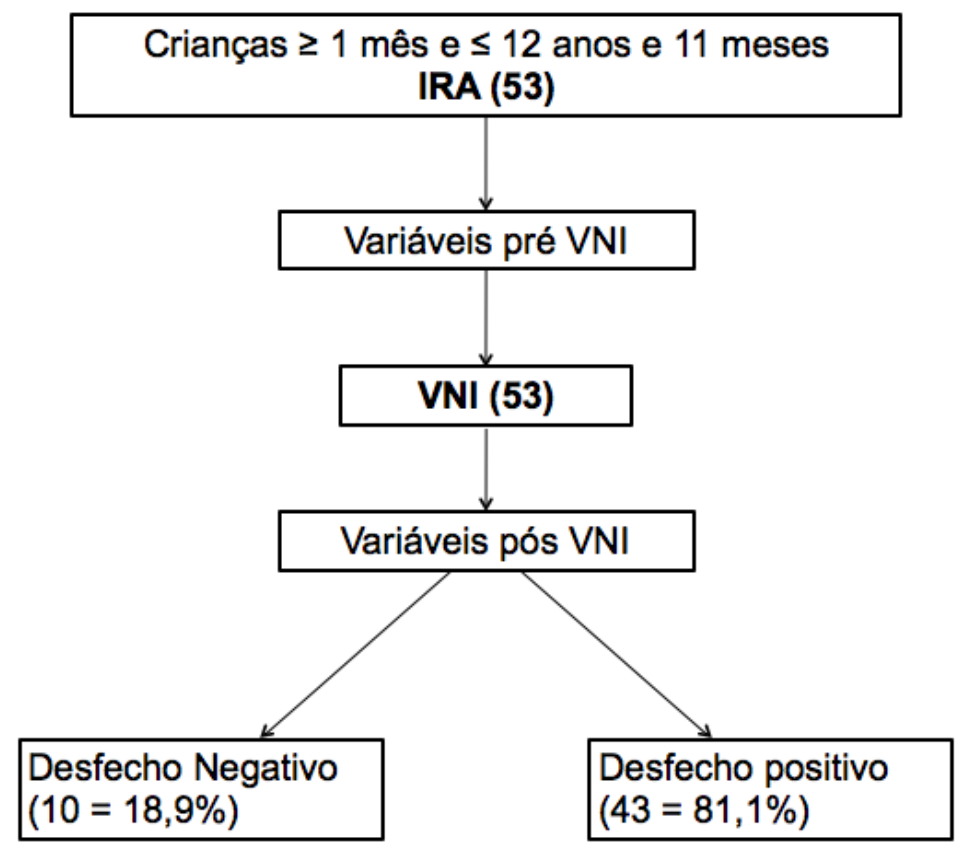

Fonte: Leite TC, et al., 2020.

A análise univariada das características dos pacientes mostrou que, dos 53 pacientes, 27 (51\%) eram do sexo feminino. A mediana de idade foi 31,2 meses (2 anos e 7 meses), e em relação à cor da pele, 36 (68\%) eram pardos ou negros. Havia 21 (39,6\%) pacientes com bronquiolite, $20(37,7 \%)$ com pneumonia e 12 ( $22,7 \%$ ) com asma. Em relação à variável Dieta foram encontrados três grupos, onde no primeiro (oral) havia 28 pacientes $(52,8 \%)$, no segundo (sonda nasoenteral) 5 pacientes $(9,4 \%)$, e no terceiro (dieta suspensa) 20 pacientes $(37,7 \%)$.

Para a variável freqüência respiratória foi encontrado mediana 60 ipm (incursões por minuto) e após VNI 30 ipm, freqüência cardíaca mediana 152 bpm (batimentos por minuto) e após VNI 132 bpm, na variável categórica Saturação mediana de $93 \%$ foi encontrada e após VNI ocorre mudança para $98 \%$. A regressão logística univariada mostrou que a cada aumento de 1 mês na idade do paciente, a chance de desfecho positivo aumenta em $2 \%$.

Não houve diferença significativa no risco de desfecho positivo quando se compara pacientes do sexo feminino e masculino, bem como pacientes de cor/raça diferentes, ou pacientes com bronquiolite ou pneumonia em reação a pacientes com asma. No entanto, pacientes com dieta oral tiveram risco 18 vezes maior de desfecho positivo do que pacientes com dieta suspensa.

Em relação às características clínicas dos pacientes, estimou-se que a cada aumento de uma unidade da EWD pré VNI o risco de desfecho positivo diminui em $75 \%$. Além disso, cada aumento de uma unidade na frequência respiratória ou cardíaca pré VNI está associado a uma diminuição de aproximadamente $9 \%$ no risco de desfecho positivo. Quanto à interface empregada na VNI observou-se que pacientes com pronga nasal tiveram risco $84 \%$ menor de desfecho positivo, enquanto que a cada aumento de uma unidade na saturação O2 pós VNI o risco de desfecho positivo aumenta três vezes seu valor (Tabela 1). 
Tabela 1 - Análise univariada dos fatores associados ao desfecho positivo.

\begin{tabular}{|c|c|c|c|c|c|}
\hline Variáveis & n (\%) $)^{*}$ & mediana $(\mathrm{AIQ})^{\star \star}$ & Razão de chance & IC 95\% & valor-p ${ }^{\star \star \star}$ \\
\hline \multicolumn{6}{|l|}{ Sexo } \\
\hline Masculino & 26 (49\%) & & 1 & & \\
\hline Feminino & 27 (51\%) & & 1,05 & $0,26-4,28$ & 0,94718 \\
\hline Idade (meses) & & $31,2(4,6-76,1)$ & 1,02 & $1,002-1,052$ & 0,0657 \\
\hline \multicolumn{6}{|l|}{ Cor } \\
\hline Branco & $17(32 \%)$ & & 1 & & \\
\hline Pardo/Negro & $36(68 \%)$ & & 0,89 & $0,17-3,74$ & 0,876 \\
\hline \multicolumn{6}{|l|}{ Diagnóstico } \\
\hline Pneumonia & $20(37,7 \%)$ & & 0,51 & $0,02-4,63$ & 0,586 \\
\hline Bronquiolite & $21(39,6 \%)$ & & 0,23 & $0,01-1,60$ & 0,1979 \\
\hline Asma & $12(22,7 \%)$ & & 1 & & \\
\hline \multicolumn{6}{|l|}{ Dieta } \\
\hline Oral & $28(52,8 \%)$ & & 18 & $2,86-353,8$ & 0,0096 \\
\hline $\begin{array}{l}\text { Sonda nasoen- } \\
\text { teral }\end{array}$ & $5(9,4 \%)$ & & 2,7 & $0,32-57,4$ & 0,4167 \\
\hline $\begin{array}{l}0 \text { (dieta sus- } \\
\text { pensa) }\end{array}$ & $20(37,8 \%)$ & & 1 & & \\
\hline $\begin{array}{l}\text { Escala Wood } \\
\text { Downes, pré VNI }\end{array}$ & & $9(7-9)$ & 0,253 & $0,006-0,598$ & 0,0117 \\
\hline $\begin{array}{l}\text { Frequência } \\
\text { respiratória (IPM), } \\
\text { pré VNI }\end{array}$ & & $60(40-68)$ & 0,91 & $0,84-0,96$ & 0,00474 \\
\hline $\begin{array}{l}\text { Frequência } \\
\text { cardiaca (BPM), } \\
\text { pré VNI }\end{array}$ & & $152(140-165)$ & 0,92 & $0,87-0,97$ & 0,00466 \\
\hline $\begin{array}{l}\text { Saturação } \mathrm{O}_{2} \text {, pré } \\
\text { VNI }\end{array}$ & & $93(90-95)$ & 1,55 & $1,16-2,27$ & 0,00816 \\
\hline \multicolumn{6}{|l|}{$\begin{array}{l}\text { Modo } \\
\text { VNI/parâmetros }\end{array}$} \\
\hline $\begin{array}{l}\text { simv/pcv } \\
\text { não simv/pcv }\end{array}$ & $\begin{array}{l}42(79,2 \%) \\
11(20,8 \%) \\
\end{array}$ & & $\begin{array}{c}0,37 \\
1\end{array}$ & $0,02-2,32$ & 0,3678 \\
\hline \multicolumn{6}{|l|}{ Interface VNI } \\
\hline $\begin{array}{l}\text { Orofacial } \\
\text { Pronga nasal }\end{array}$ & $\begin{array}{l}42(79,2 \%) \\
11(20,8 \%) \\
\end{array}$ & & $\begin{array}{c}1 \\
0,16 \\
\end{array}$ & $0,03-0,73$ & 0,0182 \\
\hline $\begin{array}{l}\text { Escala Wood } \\
\text { Downes, pós VNI }\end{array}$ & & $4(3-6)$ & 0,306 & $0,07-0,54$ & 0,00557 \\
\hline $\begin{array}{l}\text { Frequência } \\
\text { respiratória (IPM), } \\
\text { pós VNI }\end{array}$ & & $30(25-42)$ & 0,87 & $0,79-0,93$ & $<0,001$ \\
\hline $\begin{array}{l}\text { Frequência } \\
\text { cardíaca (BPM) } \\
\text { pós VNI }\end{array}$ & & $132(120-150)$ & 0,78 & $0,51-0,89$ & 0,0356 \\
\hline $\begin{array}{l}\text { Saturação } \mathrm{O}_{2}, \\
\text { pós VNI }\end{array}$ & & $98(96-98)$ & 2,98 & $1,81-7,29$ & $<0,001$ \\
\hline
\end{tabular}

Legenda: IC, intervalo de confiança. ${ }^{*} n$ (número absoluto de indivíduos) e \% (distribuição percentual dos indivíduos) foram calculados para variáveis categóricas. ${ }^{* *}$ mediana e AIQ (amplitude inter-quartílica) foram calculados para variáveis numéricas. ${ }^{* * *}$ valor-p do teste de Wald da regressão logística binária univariada. Fonte: Leite TC, et al., 2020.

Além da análise univariada, foi empregada regressão logística multivariada para avaliar os fatores associados ao desfecho positivo. Após um procedimento de seleção de variáveis, foram significativas no modelo final os seguintes fatores associados ao desfecho positivo: Idade e EWD pré VNI. As estimativas de razão de chances obtidas foram similares aquelas encontradas na análise univariada (Tabela 2). 
Tabela 2 - Modelo final de regressão logística multivariada dos fatores associados ao desfecho positvo.

\begin{tabular}{lccc}
\hline Variáveis & Razão de chance & IC 95\% & valor-p \\
\hline Intercepto & - & - & 0,00625 \\
Idade (meses) & 1,03 & $1,006-1,072$ & 0,04367 \\
Downes pré & 0,18 & $0,038-0,512$ & 0,00796
\end{tabular}

Legenda: IC, intervalo de confiança. Hosmer-Lemeshow $\mathrm{xc}^{2}=4,89 \mathrm{GL}=8$, valor- $\mathrm{p}=0,7693$. Fonte: Leite TC, et al., 2020.

Os fatores associados ao desfecho positivo no modelo final, de regressão logística multivariada, foram idade e EWD pré VNI. Os fatores associados ao desfecho positivo são: idades mais elevadas e baixos índices de EWD pré VNI (Figura 2). Demonstrado forte associação entre as variáveis e desfecho através do modelo estatístico empregado.

Figura 2 - Gráficos da probabilidade (predita pelo modelo) de desfecho positivo (a) versus a idade de um paciente com Downes pré $=9$ e (b) versus o Downes pré VNI de um paciente com idade $=31,2$ meses.

\section{(a)}

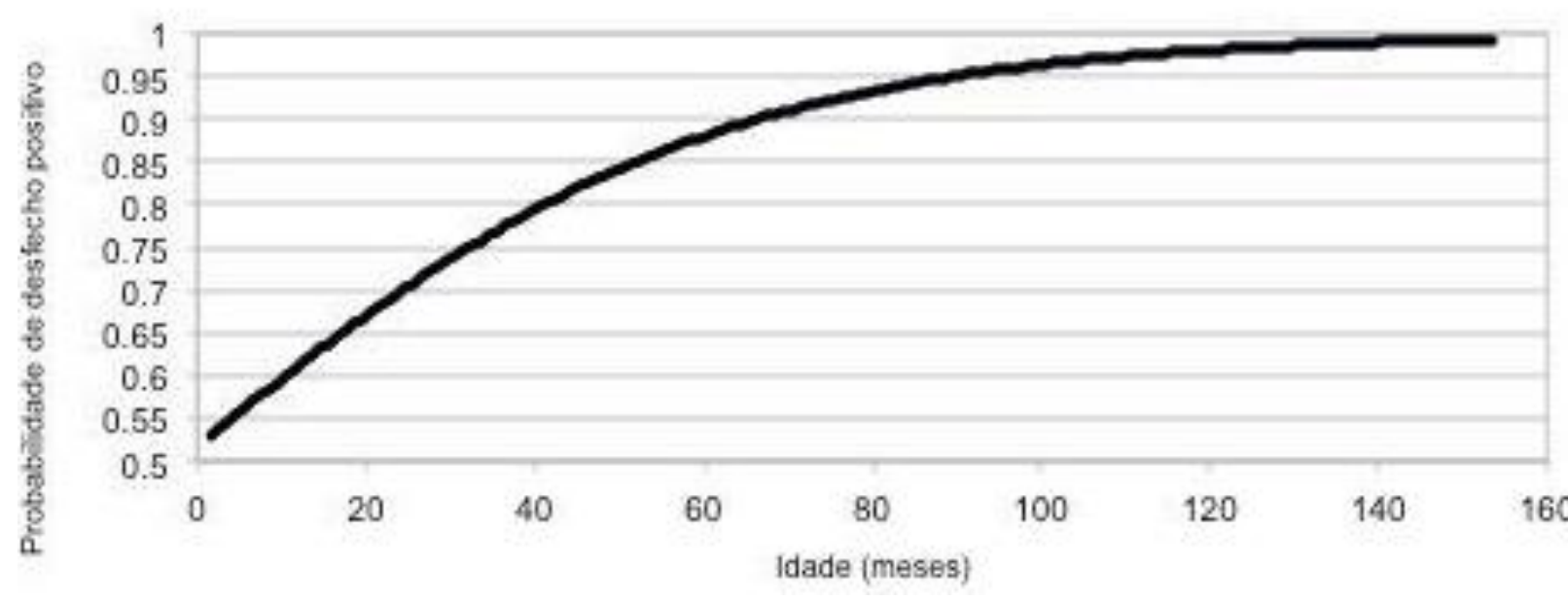

(b)

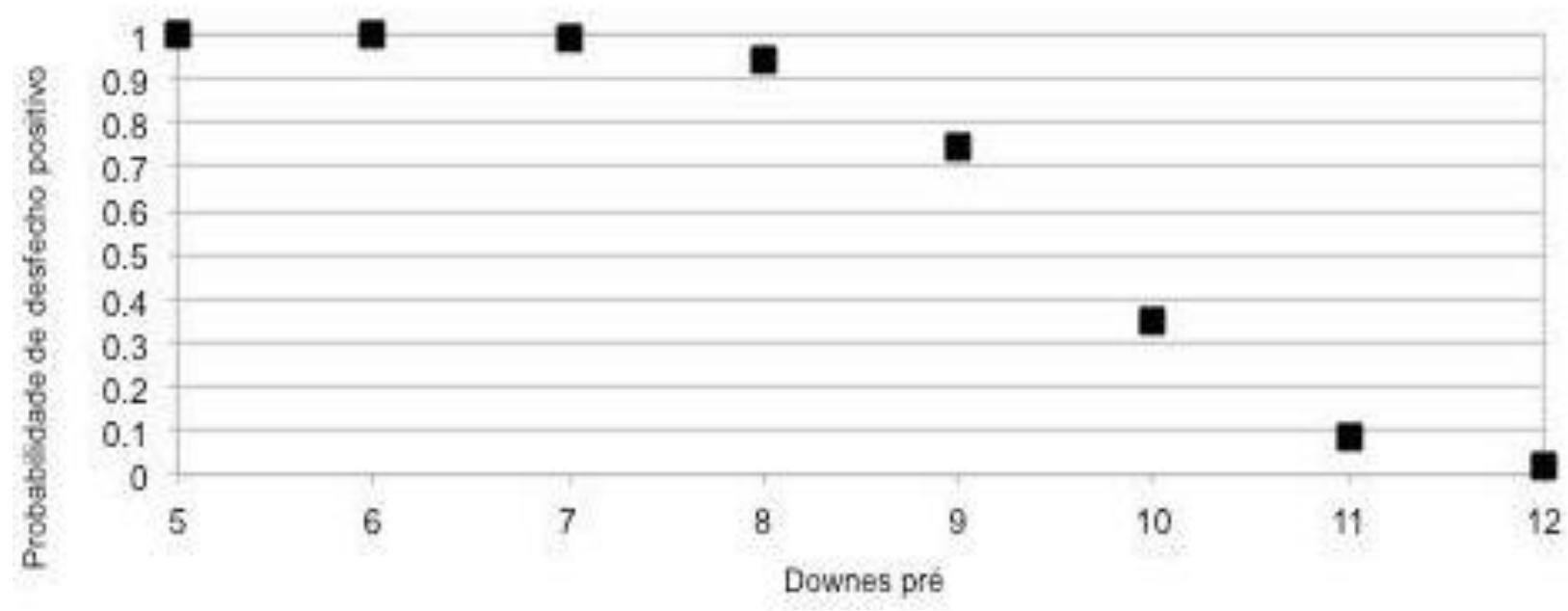

Fonte: Leite TC, et al., 2020. 


\section{DISCUSSÃO}

No presente estudo houve predomínio de pardos e negros. Este achado pode ser atribuído às diferenças sócio-econômicas desfavoráveis desses indivíduos em relação aos brancos existente no Brasil e, especificamente, no Rio de Janeiro (OSORIO RG, 2003). No local de estudo, prevalecem famílias de baixa renda e negros, segundo dados do Instituto Brasileiro de Geografia e Estatística (IBGE, 2018).

A mediana de idade de 31,2 meses do presente estudo, estaria de acordo com a literatura, uma vez que pacientes de baixa idade são mais susceptíveis à IRA (ODENA PM e CAMBRA LASAOSA FJ, 2003). A idade apresentou forte correlação com o desfecho, sendo observada na regressão logística univariada. Assim, o grupo de pacientes de baixa idade apresentou maior probabilidade de desfecho negativo. Também se observou neste estudo uma correlação com a idade e o grupo de bronquiolite viral aguda (BVA), caracteristicamente de crianças pequenas (WEINER D, et al., 2018).

A pneumonia adquirida na comunidade (PAC) ocorreu em cerca de $38 \%$ da casuística e a maior parte dos pacientes submetida a $\mathrm{VNI}$ apresentou desfecho positivo. Apenas $3 / 10$ casos apresentaram desfecho negativo. A estratégia para o manejo respiratório do paciente com PAC tem sido uma questão controversa (SCHNEIDER e SWEBERB T, 2013).

As limitações tecnológicas dos ventiladores e das interfaces utilizadas em crianças pequenas dificultam a aplicação da VNI, podendo justificar também seu desfecho negativo (ODENA PM e CAMBRA LASAOSA FJ, 2003).

A VNI por alto fluxo ou Hight Flow Nasal Cannula (HFNC), é uma opção segura, eficaz e viável para crianças com doenças respiratórias para reduzir a permanência no leito dos hospitais. Formas aprimoradas para identificar falhas de VNI- HFNC seriam benéficas para estes grupos (HOFFMAN E, et al., 2019).

Encontrados resultados diferentes do presente estudo, no qual a maior parte dos pacientes com PAC submetidos a VNI teve desfecho positivo; houve insucesso da VNI na PAC em 55\% dos pacientes e elevada mortalidade destes (SCHNEIDER J e SWEBERB T, 2013).

No presente estudo, a regressão logística univariada mostrou poucos casos de desfecho negativo, isto é $10 / 53(18,9 \%)$ casos, cuja maioria (60\%) tinha BVA. Quando comparados com pacientes com asma, o diagnóstico de BVA teve $77 \%$ menos risco de desfecho positivo. Cabe lembrar que pacientes com asma tinham dados mais elevadas, e assim, menor probabilidade de desfecho negativo. Segundo Mortamed G, et al. (2017); o benefício da VNI é significativo em algumas situações como a BVA, porém questionável em outras.

Pacientes com asma corresponderam a $22,7 \%$ dos casos, dos quais $10 \%$ tiveram desfecho negativo. No presente estudo a VNI mostrou boa resposta em pacientes com asma, corroborando estudos de Abramo T, et al. (2017); que mostraram que a pressão positiva nas vias aéreas (BiPAP) em crianças com asma na fase aguda, pode ser um tratamento eficaz. O BiPAP funciona diminuindo a sobrecarga de músculos inspiratórios fadigados, leva a efeito direto de broncodilatação, compensando a PEEP intrínseca e recrutando alvéolos colapsados. Infelizmente, em emergências pediátricas o BiPAP é subutilizado.

Quando a asma é comparada com outras doenças respiratórias em crianças não apresenta resultado significativo para desfecho de VNI. Neste estudo, quando asma foi comparada com BVA foi observado risco $77 \%$ menor de desfecho positivo para este ultimo ( $p$ valor $=0,1979$ ). Equivalendo- se ao estudo de Abramo T, et al. (2017); no entanto, quando se compara asma e PAC não há significância estatística.

No presente estudo houve associação de pacientes com EWD mais baixos com desfecho positivo. $\mathrm{Na}$ análise univariada foi verificada a mediana da EWD pré VNI $=9(9-9,8)$, nos pacientes que evoluíram para macronebulização/ aa enquanto que a mediana de EWD pré VNI $=8$ (7-9) naqueles que evoluíram para ventilação mecânica invasiva ( $p$ valor 0,001 ). A EWD pré VNI demonstrou forte associação com o desfecho positivo: menores índices de EWD pré VNI tenderam ao desfecho positivo. Houve predominância na população de estudo de desfecho positivo, com EWD pós VNI mais baixos, mesmo naqueles com valores de 
EWD pré VNI mais elevados, demonstrando respostas positivas com o método não invasivo. Na literatura foi demostrado em vários estudos que a avaliação clínica feita de forma adequada facilita o tratamento e o planejamento terapêutico do paciente melhorando a qualidade no cuidado e no manejo destes. A VNI, é atualmente o tratamento de primeira linha para IRA uma vez que está associada à redução na taxa de intubação (BARBAS CS, et al., 2014; ANTONELLI M, et al., 2001; NUNES P, et al., 2010).

Autores estudaram modos de avaliação do paciente pediátrico com IRA e observaram que a EWD tem boa correlação com a condição clínica de crianças com crise asmática. A EWD foi validada por consenso e baseia-se nos mecanismos de compensação ativados antes da dificuldade respiratória devido à obstrução da via aérea. Sua principal força é incluir sintomas clínicos. Isso também fica evidente quando há uma boa correlação com saturação de oxigênio e seu uso na clínica poderia permitir padronizar avaliações em qualquer lugar onde os pacientes são tratados (GARCÍA-SOSA A, et al., 2018).

Algumas escalas usam apenas variáveis clínicas, mas outros se baseiam na saturação de oxigênio, pico de fluxo expiratório, testes de função pulmonar, frequência respiratória, freqüência cardíaca, $\mathrm{PaO} 2$ e PaCO2, ou combinações (GARCÍA-SOSA A, et al., 2018; TANAKA N, et al., 2015). A EWD foi de melhor desempenho. A aplicação rotineira da EWD poderia unificar as avaliações e possíveis tratamentos na IRA.

No presente estudo a SatO2 foi outra variável de grande correlação com o desfecho positivo, corroborando com estudos encontrados (LOPEZ-FERNANDEZ Y, et al., 2012). Esta variável foi útil na avaliação dos pacientes, constatando-se que a cada aumento de uma unidade na saturação pós $\mathrm{VNI}$, o risco de desfecho positivo aumenta três vezes seu valor ( $p$ valor 0,001 ). A freqüência respiratória também presente na EWD, foi outra variável que demonstrou forte associação ao desfecho ( $p$ valor 0,001 ), sendo os valores de freqüência respiratória mais baixos tanto pré quanto pós $\mathrm{VNI}$ associados com desfecho positivo.

Outra variável avaliada foi a frequência cardíaca demonstrando também associação com o desfecho, porém em menor valor que a freqüência respiratória. A cada aumento de 1 unidade na frequência cardíaca pós $\mathrm{VNI}$, o risco de desfecho positivo diminui em $22 \%$, com intervalo de confiança de $11 \%$ a $49 \%$. (p valor $0,0356)$. Na literatura foi demonstrado para caso de insucesso de VNI a freqüência cardíaca em valores muito baixos pós aplicação da técnica terapêutica, ou seja, bradicardia devido a diminuição de retorno venoso, não ocorrendo em nosso estudo (PANTONI CBF, et al., 2009; FERREIRA LL, et al., 2014). Segundo Pons-Ódeña $\mathrm{M}$, et al. (2019); pacientes com maiores frequências cardíacas e maior pressão positiva inspiratória nas vias aéreas (fornecida pela VNI) em 2 horas pós aplicação da técnica, apresentaram maior risco de falha ou seja, desfecho negativo, à semelhança do presente estudo.

Quanto à dieta, pacientes com dieta oral tiveram risco 18 vezes maior de desfecho positivo. A autonomia da criança (alimentação sem intercorrências ou manutenção das atividades de vida diária) tem correlação direta com um quadro de IRA mais branda (VICTORA CG, et al., 1999). Não foram encontrados estudos que avaliem a correlação da dieta com a VNI. Não encontramos diferença significativa no risco de desfecho positivo quando se compara os grupos de pacientes com dieta por sonda nasoenteral com pacientes com dieta suspensa, no entanto o desfecho foi igual para ambos os grupos ( $p$ valor. 0,0096 ).

Nossos pacientes com pronga nasal tiveram risco $84 \%$ menor de desfecho positivo, quando comparados aos com interface orofacial. Mesmo que a maior parte da população estudada tenha feito uso da interface orofacial a maior parte da pequena amostra que utilizou pronga nasal evoluiu para desfecho negativo. Além disso, houve também correlação com a baixa idade e com o diagnóstico de BVA. Este aliás, foi o diagnóstico principal dos pacientes que usaram pronga nasal (90\%), confirmando achados acima citados, sugerindo que a imaturidade pulmonar característica dos pacientes com menor idade e a dificuldade de adaptação a interface, são fatores que desafiam o bom desfecho pós aplicação de VNI nesta faixa etária (NAVALESI P, et al., 2000).

Segundo Navalesi P, et al. (2000) ; embora o uso de máscara orofacial aumente o risco de broncoaspiracão e facilite a distensão gástrica, seu uso é geralmente o mais recomendado em pacientes com IRA. A aplicação da mascara nasal e da facial tem resultados semelhantes no desfecho clinico de pacientes em VNI, embora a primeira seja melhor tolerada em adultos (KWOK H, et al., 2003). 
O modo ventilatório não foi uma variável significativa. Poucos estudos abordam observações sobre modo ventilatório em VNI especificamente. Os modos ventilatórios setados para VMI independente de ser invasiva ou não, mostram que os aparelhos de ventilação mecânica modernos apresentam mecanismos de compensação para assincronia, corroborando com estudos encontrados (SCHETTINO GPP, et al., 2007). Assim, o que os estudos possivelmente mostram são as limitações tecnológicas e de aplicação dos aparelhos utilizados, e nem tanto se o modo ventilatório pode ser um verdadeiro preditor.

Observado que escolha correta da interface levando em consideração o tamanho e material, assim como sua boa aplicação inicial são de fundamental importância para o sucesso da VNI; sugerindo mais estudos em relação as interfaces, afim de facilitar desfechos favoráveis ás crianças que deste método necessitam.

Houve forte significância estatística para correlação de idade e EWD com o desfecho, como demonstrado nos gráficos expressos nas figura 2. Fatores associados ao maior risco de desfecho positivo foram: idades maiores, e menores índices de EWD pré VNI, corroborando com estudos. Foi constatado que, estes achados demonstraram fatores importantes para observação de um bom desfecho em crianças submetidas a VNI por IRA (JOSHI G e TOBIAS JD, 2007).

\section{CONCLUSÃO}

Foram encontrados fatores associados ao desfecho positivo: idades maiores e menores índices de EWD pré VNI, sendo este método de avaliação um importante preditor para sucesso da terapêutica utilizada. A freqüência respiratória foi um importante sinal clínico para tomada de decisão na continuidade ou não da VNI. Foi constatado que a técnica de VNI contribuiu de forma positiva com a população estudada e o EWD foi importante instrumento de avaliação para o momento e escolha da técnica de VNI empregada.

\section{REFERÊNCIAS}

1. ABRAMO T, et al. Programa pediátrico de melhoria contínua da qualidade do ED BiPAP com análise do paciente: 2005-2013. BMJ open. 2017; 7: 1-13.

2. ANTONELLI M, et al. Predictors of failure of noninvasive positive pressure ventilation in patients with acute hypoxemic respiratory failure: a multicenter study. Intensive Care Med. 2001; 27: 1718-1728.

3. BARBAS CS, et al. Recomendações brasileiras de ventilação mecânica. Rev Bras Ter Intensiva. 2014; 26(2): 89-121.

4. FERREIRA LL, et al. Efeitos da ventilação mecânica não invasiva sobre a modulação autonômica cardíaca, Rev Bras Cardiol. 2014; 27(1): 53-58.

5. FONSECA JG, et al. Avaliação e manejo inicial da insuficiência respiratória aguda na criança. Rev Med Minas Gerais 2013; 23(2): 2 .

6. GARCÍA-SOSA A, et al. Escala Wood Downes-Ferrés, una opción útil para identificar la gravedad en crisis asmática. Rev Mex Pediat, 2018; 85(1): 11-16.

7. GARCÍA-SOSA A, et al. Escala Wood Downes-Ferrés, una opción útil para identificar la gravedad en crisis asmática. Rev Mex Pediat, 2018; 85(1):18-22.

8. GONZAGA CS, et al. Ventilação não invasiva em crianças com insuficiência respiratória aguda: uma revisão sistemática. Einstein. 2011; 9(1 Pt 1): 90-4.

9. HAMMER J. Acute respiratory failure in children. Ped Resp Rev. 2013; 14: 64-69.

10. HOFFMAN E, et al. A review of the use of high-flow nasal cannula oxygen therapy in hospitalised children at a regional hospital in the Cape Town Metro, South Africa. S Afr Med J. 2019; 109(4): 272-277.

11. HOLANDA MA, et al. Ventilação não-invasiva com pressão positiva em pacientes com insuficiência respiratória aguda: fatores associados à falha ou ao sucesso. J. Pneumologia. 2001; 27(6): 301-309.

12. JOSHI G, TOBIAS JD. A five-year experience with the use of BiPAP in a pediatric intensive care unit population. J Intensive Care Med. 2007; 22: 38-43.

13. KWOK H, et al. Controlled trial of oronasal versus nasal mask ventilation in the treatment of acute respiratory failure. Crit Care Med. 2003;31(2):468-73.

14. LOPEZ-FERNANDEZ Y, et al. Estudo de Epidemiologia e História Natural da Lesão Pulmonar Aguda Pediátrica: incidência e desfecho da síndrome do desconforto respiratório agudo em crianças. Crit Care Med. 2012; 40: 32383245.

15. MORTAMET G, et al. Interfaces for noninvasive ventilation in the acute settings in children. Paediatr Respir Rev 2017; 23: 84-8.

16. MUÑOZ-BONET JI, et al. Noninvasive ventilation in pediatric acute respiratory illness, Clinical Pediatric Emergency Medicine, World J Pediatr. 2010; 6(4): 323-330.

17. NAVALESI $P$, et al. Physiologic evaluation of noninvasive mechanical ventilation delivered with three types of masks in patients with chronic hypercapnic respiratory failure. Crit Care Med. 2000; 28: 1785-90. 
18. NUNES $P$, et al. Ventilação não invasiva numa unidade de cuidados intensivos pediátricos. Acta Med Port. 2010;23(3):399-40.

19. ODENA PM, CAMBRA LASAOSA FJ. Mechanical ventilation in pediatrics (III). Weaning, complications and other types of ventilation. Noninvasive ventilation. Sociedad Española de Cuidados Intensivos Pediátricos. An Pediatr.(Barc-Spanish). 2003;59(2):165-72.

20. OSORIO RG. O sistema classificatório de cor ou raça do IBGE (Instituto brasileiro de Geografia e estatística). IPEA (Instituto de Pesquisa Econômica Aplicada) 2003; 996:1415-4765.

21. PANTONI CBF, et al. Acute application of bilevel positive airway pressure influences the cardiac autonomic nervous system. Clinics (São Paulo). 2009;64(11):1085-92.

22. PONS-ÒDENA M, et al. ¿Cuáles son los factores predictores de fracaso de ventilación no invasiva más fiables en una unidad de cuidados intensivos pediátricos? An Pediatr (Barc). 2019; ISSN: 1695-4033. DOI: 10.1016/j. anpedi. 2019.01.013.

23. POPAT B, JONES AT. Invasive and non-invasive mechanical ventilation. Medicine (Baltimore) 2012; 40(6):298.

24. P.V. (Prosthesis Ventilatory). MAQUET Critical Care Röntgenvägen 2, Solna - Suécia SE- 171- 95.

25. R CORE TEAM. A language and environment for statistical computing. $r$ foundation for statistical computing, Viena, Austria, 2017.

26. https://www.r-project.org/

27. RIMENSBERGER PC. Noninvasive pressure support ventilation for acute respiratory failure in children. Schweiz Med Wochenschr. 2000;130(49).

28. SCHETTINO GPP, et al. III Consenso Brasileiro de Ventilação Mecânica. J Bras de Pneumol. 2007; 33(Supl 2):92105.

29. SCHNEIDER J, SWEBERB T. Acute Respiratory Failure. Crit Care Clin. 2013; 29:167-183.

30. SMITH A, et al. Initiation of Noninvasive Ventilation for Acute Respiratory Failure in a Pediatric Intermediate Care Unit. Hosp Pediatr. 2019;9(7):538-544.

31. SOCIEDADE BRASILEIRA DE PEDIATRIA. Insuficiência Respiratória Aguda. Departamento Científico de Terapia Intensiva; 2017. n.2.

32. TANAKA N, et al. Community acquired pneumonia: a correlative study between chest radiographic and HRCT findings. Jpn J Radiol. 2015;33(6):317-28.

33. TOBIN MJ. Culmination of an era in research on the acute respiratory distress syndrome. $\mathrm{N}$ Engl $\mathrm{J}$ Med. 2000;342(18):1360-1361.

34. VICTORA CG, et al. Potential interventions for the prevention of childhood pneumonia in developing countries: improving nutrition. Am J Clin Nutr. 1999;70(3):309-320.

35. WEINER D, et al. Causes of acute respiratory distress in children. 2018. Available from: https://www.uptodate.com/contents/causes-of-acute-respiratory-dis-tress-in-children. 